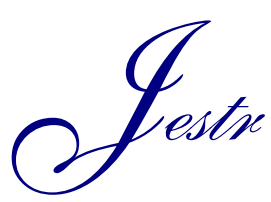

\title{
Simulation of a Compressible Powder Flow under Oscillatory Shear Stress Modeled as a Non-Linear Fluid by Using an Explicit Solution Method
}

\author{
Mauricio E. Robledo ${ }^{1, *}$, Luis G. Obregón ${ }^{2}$ and Carlos Velázquez ${ }^{3}$ \\ ${ }^{1}$ Research group on process simulation and coal transformation, Chemical Engineering Program, Universidad del Atlántico, Carrera \\ 30 Número 8-49 Puerto Colombia - Atlántico, 081007, Colombia. \\ ${ }^{2}$ Research group on sustainable chemical and biochemical processes, Chemical Engineering Program, Universidad del Atlántico, \\ Carrera 30 Número 8- 49 Puerto Colombia-Atlántico, 081007, Colombia. \\ ${ }^{3}$ Chemical Engineering Department, University of Puerto Rico, Mayagüez Campus, PO Box 9000, Mayagüez PR 00681.
}

Received 26 March 2017; Accepted 4 September 2018

\begin{abstract}
The purpose of this research is to develop the modeling and simulation of a frictional and compressible powder flow subjected to oscillatory shear stress in a device that consists of a rectangular shaped cavity with a free surface, two oscillatory moving walls, and three non-moving walls. The finite differences method under the explicit scheme is used to approximate the real solution to the resulting equations from the mathematical modeling of the studied system. The simulation results were compared qualitatively with experimental results, and the solution method was verified by comparing the simulation results of a viscous glycerin flow with the results of the software STAR-CCM+ under the same conditions of the powder flow. The results showed that the powder velocity profiles were coherent with the experimental results. Furthermore, the solution method was validated finding that the $83.5 \%$ of the compared data presented absolute errors less than $0.5 \mathrm{~cm} / \mathrm{s}$. The complete velocity profiles obtained from the simulation help to have a deep understanding of the granular material behavior subjected to oscillatory shear stress.
\end{abstract}

Keywords: Powder Flow, Granular Material, Shear Stress, Continuum Mechanic, Modeling, Simulation.

\section{Introduction}

The development of mathematical models for the simulation and research of granular material flow is a relevant topic in engineering processes that comprehends a wide range of industries, such as pharmaceutical, chemical, foods, plastics, advanced material, etc., [1]. When it is produced a drug in a pharmaceutical industry, it is well known that small changes in the properties of the ingredients or in the operating conditions of a process can cause a negative impact on its quality, leading to dangerous and expensive consequences. The fact that just a small change in the operating parameters can result in a final product with unpredictable physicochemical properties, has provoked the use of exhaustive monitoring systems in pharmaceutical companies in order to control and make the product quality as efficient as possible [2]. Therefore, the understanding of the physical phenomena that govern the flow and the mixing process of the ingredients that participate in the production of medicines is extremely important to guarantee their quality and to minimize health risks [3].

There is a large amount of information about the flow and mixing of fluids that lack fundamental theories and engineering experiences necessary to design reliable and scalable processes to manufacture products made of granular materials. This situation happens due to the high complexity of the granular material behavior [4]. This implies that the

*E-mail address: mrobledo@mail.uniatlantico.edu.co

ISSN: $1791-2377 @ 2018$ Eastern Macedonia and Thrace Institute of Technology. All rights reserved. doi:10.25103/jestr.114.11 design and scaling of such processes were made using empiricism and observation instead of theoretical methods $[5,6]$. Companies avoid making changes in their manufacture processes due to the mentioned reason.

Depending on the concentration of actives ingredients, the units employed for powder mixing are based on different transport mechanisms, such as diffusion, and convection [7]. Currently, the predictions about the behavior of powder are based on continuum mechanics [8-18] and discrete element methods $[1,19]$. The latter has the constraint that requires a high computational power when the number of particles in the system is excessively high. The second offer a very good alternative solution to study and simulate the powder behavior. However, most of the solutions have been found only in two dimensions [12] due to the complexity of the equations with no experimental work to compare and validate. For instance, Fernandez et al. [20], studied granular flow in 2D with a well-balanced discretization of the proposed multilayer shallow model. Zhao and Massoudi [21], studied the flow of granular materials between two shearing plates, subjected to slip at the walls. Abrahamsson et al. [8] studied the continuum modeling of dense granular flow in a Couette shear cell in 2D.

In this work, it will be presented the simulation made of the flow of granular materials at different operating conditions in a mixing device that consisted of an open box with two moving walls and three static ones [4,22]. The motion of the powders is caused by the shear stress applied by the moving walls to the particles. With the use of the continuum mechanics and the best chosen constitutive equation reported in the literature for the regime of flow 
called quasi-static, slow or frictional [23], a 3D simulation was run and then compared with experimental results obtained in the mentioned mixing device. The goals were to demonstrate the importance of considering the third dimension and to gain insights of what physical phenomena are really involved in the 3D granular flow. Because most of the powder mixers rely on shear stress, it was chosen to work in rectangular coordinates in the mixing device. It provides useful information suitable for interpreting powder flow in many types of mixers.

\section{Materials and Methods}

\subsection{System of Study}

An amount of granular material was contained in a rectangular shaped cavity such as shown in Fig. 1. First, the solid was in repose, then the two parallel walls to the $\mathrm{XZ}$ plane start to move oscillatory. The first wall moves in $\mathrm{X}$ positive direction with $v_{\text {wall }}$ velocity and the second wall, parallel to the first, moved at the same velocity but in the opposite direction, both of them during a time $\mathrm{T} / 2$. Right after, the walls changed the movement direction until a complete period $\mathrm{T}$.

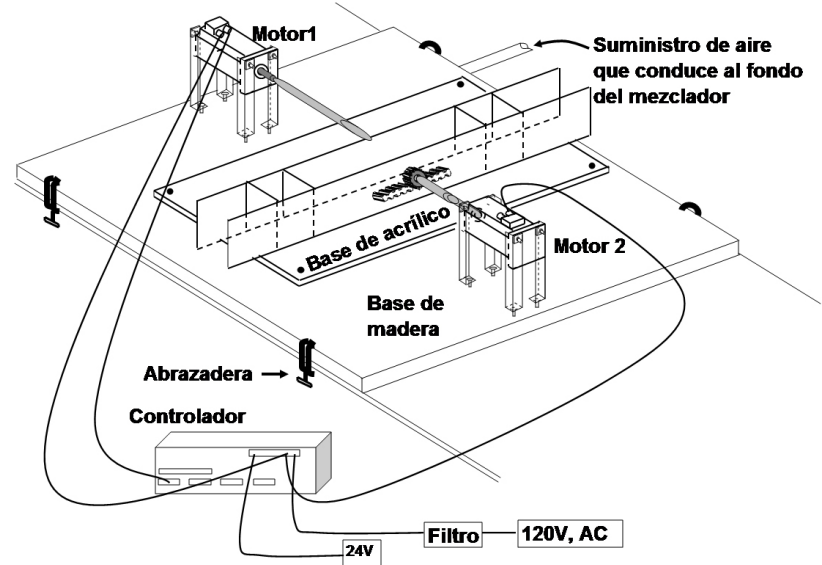

Fig. 1. Schematic of the oscillatory mixer

Fig. 2, shows the movement of the walls with velocity in the $\mathrm{X}$ direction as a function of time according to the following equation:

$v_{\text {wall }}=\left\{\begin{array}{rc}v_{0} & \text { si } 0<t[s]<T / 2 \\ -v_{0} & \text { si } T / 2<t[s]<T\end{array}\right.$

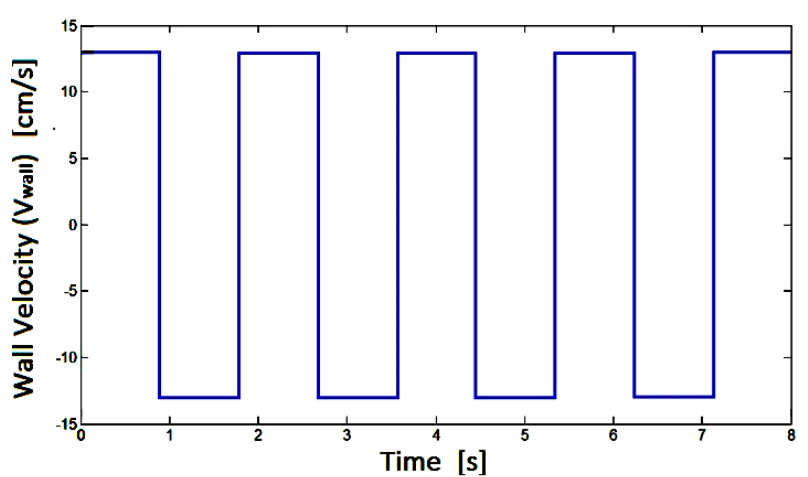

Fig. 2. Velocity of the walls
The cavity bottom was static, and the solid particles on the surface were in contact with air; therefore, they slide freely.

The dimensions of the oscillatory mixer and physical properties of the granular material are shown in Tab. 1 and Tab. 2, respectively.

Table 1. Mixer dimensions

Dimensions

\begin{tabular}{l|r|l}
\hline Width (W) in X - Direction & 2.79 & $\mathrm{~cm}$ \\
\hline Length (L) in Y - Direction & 22.36 & $\mathrm{~cm}$ \\
\hline Height (H) in Z - Direction & 5.08 & $\mathrm{~cm}$ \\
\hline
\end{tabular}

Table 2. Granular material properties Properties

\begin{tabular}{l|r|l}
\hline Particle density $\left(\boldsymbol{\rho}_{\boldsymbol{s}}\right)$ & 1.4 & $\mathrm{~g} / \mathrm{cm}^{3}$ \\
\hline Internal friction angle $\left(\boldsymbol{\phi}_{\boldsymbol{s}}\right)$ & 30 & degree \\
\hline Cohesivity $(\mathbf{C})$ & 0 & \\
\hline Volumetric fraction of the particles & 0.5 & $\mathrm{~g}$ sol./g \\
$(\boldsymbol{\varepsilon})$ & 4 & tot. \\
\hline
\end{tabular}

\section{Mathematical Modeling}

The mathematical model used for the approximation of granular material behavior results from the application of the momentum and the material balance (continuity equation), using a proper constitutive equation for granular materials [24].

\subsection{Continuity equation}

The material balance describes the density fluid variation rate as a function of time at a specific point in the space. The continuity equation, in the vector system, is given by the following equation as a function of the particle volume fraction $(\varepsilon)$ :

$$
\frac{\partial \varepsilon}{\partial t}=-\nabla \cdot(\varepsilon \mathbf{v})
$$

\subsection{Momentum balance}

The following vector equation expresses the differential momentum balance:

$$
\frac{\partial \rho \mathbf{v}}{\partial t}+\nabla \cdot(\rho \mathbf{v v})=-\nabla \cdot \mathbf{T}+\rho \mathbf{g}
$$

\subsection{Constitutive equation: Plastic Potential Theory}

The Plastic Potential theory provides a way to predict the velocity distribution in a granular environment at the point in which the granular material is consolidated and starts to dilate. This theory explains the relationship between the stress tensor and the strain rate (Eq. 4) using three different theories: the yield function (Y), the plastic-potential function (G) and a flow rule [25-27].

$$
\mathbf{D}=-\frac{1}{2}\left[\nabla \mathbf{v}+\nabla \mathbf{v}^{T}\right]
$$


In the modeling process, Pitman-Schaeffer-Gray-Stiles yield function taken from Dartavelle publication is used [23]:

$$
Y=I I_{d T}+\left[(\bar{\sigma}-P)^{2}-P^{2}\right] \sin \left(\varphi_{s}\right)=0
$$

Where $I I_{d T}$ is the second invariant of the deviator frictional stress tensor $\mathbf{T}, \bar{\sigma}$ is the normal principal stress, $\sigma_{m}$ is the arithmetic average of the principal stresses, $\mathrm{P}$ is a function that measures the compressibility of the granular material and increases monotonically with the volume fraction of the particles and is an isotropic-hydrostatic normal stress. The state equation to calculate the pressure is given by the following equation, [9]:

$$
P=F r \frac{\left(\varepsilon-\varepsilon_{\min }\right)^{\eta}}{\left(\varepsilon_{\max }-\varepsilon\right)^{p}}
$$

where $F r, \eta$ and $p$ are empiric constants. In this simulation, these values were taken from Dartavelle publication, [25]: $F r=1000, \eta=p=3$. In frictional regime, pressure and granular material viscosity increase asymptotically to infinite from a volume fraction of solid of $50 \%$ vol. to $64 \%$ vol. approximately.

The strain rate at any arbitrary direction $\left(D_{m}\right)$ is proportional to the partial derivative of the Plastic Potential Function $(\mathrm{G})$ with respect to the corresponding stress $\left(\sigma_{\mathrm{m}}\right)$.

$$
D_{m}=q \frac{\partial G}{\partial \sigma_{m}}
$$

where $q$ is a positive scalar, often called plastic multiplier. This scalar is a property of the flow conditions.

The flow rule states the relationship between the functions $\mathrm{G}$ and $\mathrm{Y}$ as follows:

$$
G=Y
$$

The following vector equation is obtained from Eq. 4 and Eq. 7:

$$
\mathbf{D}=q \hat{\mathbf{T}}+\frac{2}{3} q\left(\sin ^{2}\left(\varphi_{s}\right)\right)(\bar{\sigma}-P) \mathbf{I}
$$

Where $\hat{\mathbf{T}}$ is the deviator of the shear stress tensor (i.e., pure shear stress) and $\mathbf{I}$ is the unitary tensor.

The velocity divergence $(\nabla \cdot \mathbf{v})$ is equivalent to the negative first invariant of the strain rate.

$$
\nabla \cdot \mathbf{v}=-\sum_{m=1}^{3} D_{m}=-2 q \sin ^{2}\left(\varphi_{s}\right)(\bar{\sigma}-P)
$$

An important relationship called Levy - Von Mises flow rule is obtained by combining the equations 8 and 9 , as follows [25]:

$$
\hat{\mathbf{D}}=q \hat{\mathbf{T}}
$$

The expressions for the dynamic and dilatational viscosities ( $\mu$ and $\kappa$, respectively) from the results of the Plastic Potential theory are:

$$
\mu=\frac{P \sin ^{2}\left(\varphi_{s}\right)}{\sqrt{4 \sin ^{2}\left(\varphi_{s}\right) I I_{d D}+(\nabla \cdot \mathbf{v})^{2}}}
$$

and,

$$
\kappa=\frac{P}{\sqrt{4 \sin ^{2}\left(\varphi_{s}\right) I I_{d D}+(\nabla \cdot \mathbf{v})^{2}}}
$$

In granular materials, the dynamic viscosity measures the chaotic movement of the particles and the dilatational viscosity of the effects produced by vibration and rotation of them.

The frictional stress tensor is given by:

$\mathbf{T}=P \mathbf{I}+2 \mu \mathbf{D}-\lambda \nabla \cdot \mathbf{v I}$

Where,

$$
\lambda=\kappa-\frac{2}{3} \mu
$$

So, the divergence of the frictional stress tensor is:

$$
\nabla \cdot \mathbf{T}=\nabla P+\nabla \cdot(2 \mu \mathbf{D})-\nabla(\lambda \nabla \cdot \mathbf{v})
$$

Eq. (4) is combined with Eq. (16), and some terms are grouped to yield the next equation:

$\nabla \cdot \mathrm{T}=\nabla \mathrm{P}-\nabla \cdot(\mu \nabla \mathrm{v})-\mathrm{S}_{\mathrm{M}}$

\section{Simulation}

The mathematical modeling provides a set of equations that simulate the behavior of the flow of granular material by different theories with experimental validation. In this case, the modeling result in a differential partial and not linear system of equations in the three-dimension domain that needs the use of numerical methods to find the exact solution of the system.

The simulation parameters are taken from Obregón et al., [4]; the selected velocity of the oscillatory walls ( $\left.v_{\text {wall }}\right)$ was $13 \mathrm{~cm} / \mathrm{s}$, the amplitude (A) was $22.86 \mathrm{~cm}$ and its period of oscillation was 1.76 seconds. This combination of parameters makes the powders in this system to stay in the regime of slow-frictional flow.

\subsection{The Finite Difference Method: Explicit Scheme}

The Finite Difference Method (FDM) is based on the approximation of partial derivatives using the differences between the values of a variable or function in the points of a mesh. The discretization of the model was developed using the forward difference at time $t_{n}$ (also called forward Euler method) with a second-order central difference for the space 
derivate of the variable at the position $\left({ }_{i, j, k}\right)$ to determine gradients and divergences [28].

The discretized equation of continuity at time $t_{n}$ is given by the next expression:

$\varepsilon^{n+1}=-\Delta t \nabla \cdot(\varepsilon \mathbf{v})^{n}+\varepsilon^{n}$

And, the discretized equation of momentum at time $t_{n}$ is:

$$
\mathbf{v}^{n+1}=\mathbf{v}^{n}-\frac{\Delta t}{\rho_{s} \varepsilon^{n}}\left[\rho_{s} \varepsilon^{n}(\mathbf{v} \cdot \nabla \mathbf{v})^{n}+\nabla \cdot \mathbf{T}^{n}+\rho_{s} \varepsilon^{n} \mathbf{g}^{n}\right]
$$

The velocity profiles in the $\mathrm{X}$ direction using this method is presented in Fig. 3 and Fig. 4.

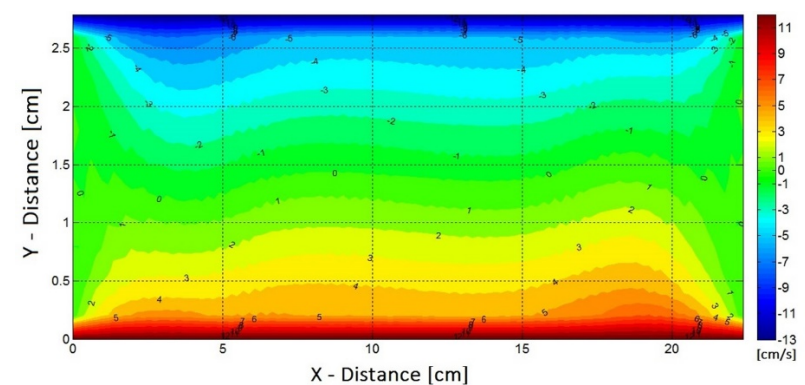

Fig. 3. $X$ Velocity field at $Z=2.92 \mathrm{~cm}$

In Fig. 3, the velocities near the moving walls decrease as they approximate to the center of the plane till reaching the value of zero. Similarly, the $X$-velocities at the proximities of the fixed walls are approximately zero. It means that the solid particles move in the direction of the moving walls suffering an abrupt change when they come to the corner of the mixer, evidently due to the collision with the static wall. It was assumed that the walls moved uniformly with a constant velocity in the boundary conditions, and the particles next to these walls moved at the same velocity, i.e., $v_{\text {wall }}= \pm 13 \mathrm{~cm} / \mathrm{s}$.

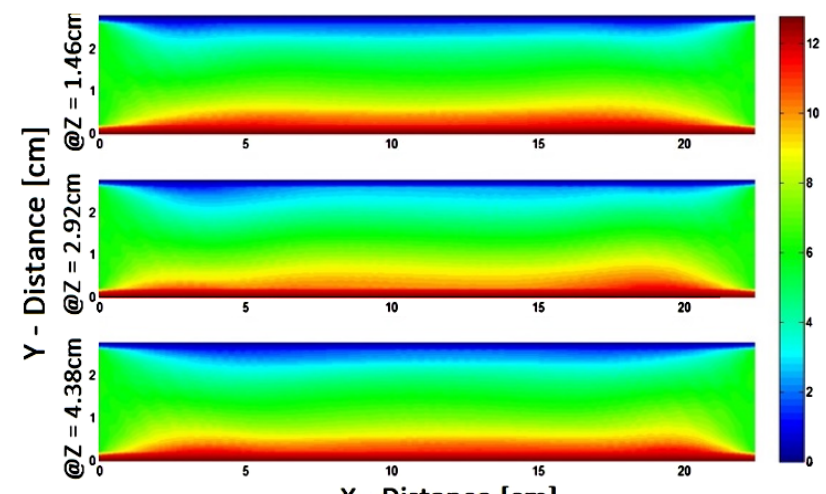

Fig. 4. Profile of the $X-V$ elocity at different heights

Furthermore, the X-velocity profiles in the XY plane had the same pattern along the $\mathrm{Z}$ direction, as shown in Fig. 4, except in the proximities to the floor of the device (@Z $Z=0$ $\mathrm{cm})$ where the velocities are zero. As soon as the solid particles next to the corners hit the static wall, they change their direction of movement. Many particles that initially were moving in the $\mathrm{X}$-direction begin to move in the $\mathrm{Y}$ direction, reaching a Y-velocity maximum in the half of the path (@Y $\mathrm{Y}=\mathrm{L} / 2)$. Also, according to Fig. 5, the Y-velocities are different to zero except in regions close to the fixed walls where they are zero due to the wa toll friction. By using a vector field with arrows of the total resultant velocity at $\mathrm{Z}=2.92$, the effects at the corner of the oscillatory mixer can be observed (see Fig. 6).

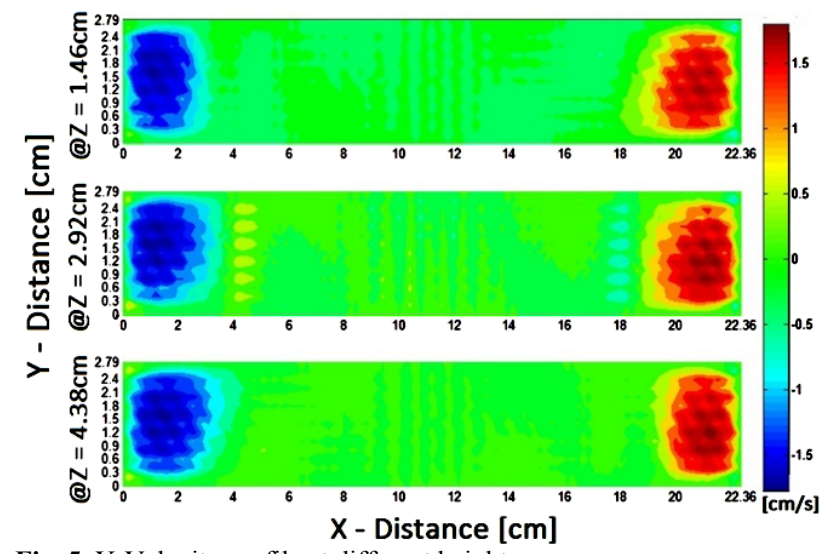

Fig. 5. Y-Velocity profile at different heights

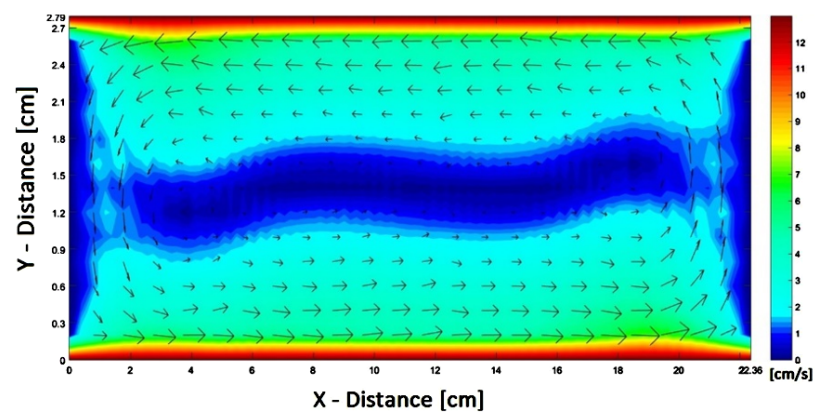

Fig. 6. Vector velocity field at $Z=2.92 \mathrm{~cm}$

Fig. 7 shows the total velocity profiles in the XY plane at different heights with almost the same pattern, low velocities in the center and high velocities in the zone near the moving walls.

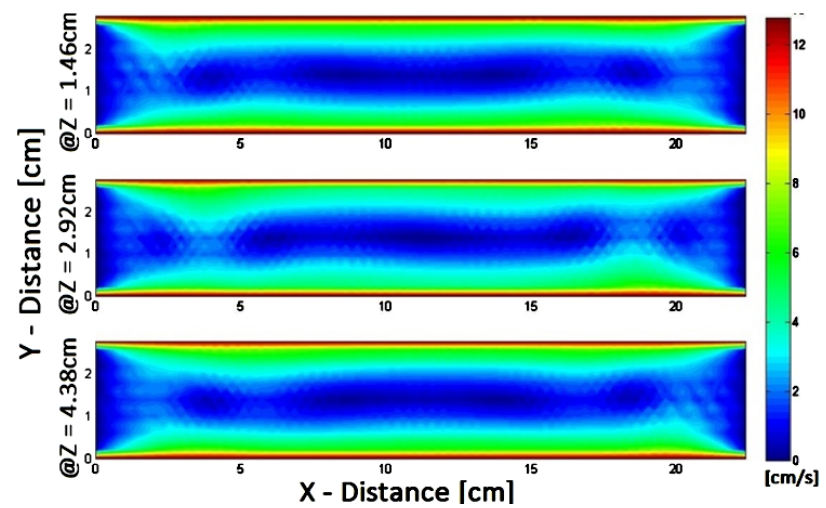

Fig. 7. Profile of the total velocity at different heights

About the Z-direction, Z-velocity profiles are shown in Fig. 8. It is possible to observe in the different planes that $Z$ velocities change their magnitude and orientation as well. This change in direction drawn with blue and red in Fig. 6, create vortexes that take place inside the mixer increasing the porosity in that zone and contributing to the particle mixing process in the $\mathrm{Z}$-direction.

The profiles of the total velocity in the $\mathrm{YZ}$ plane are shown in Fig. 9, where the velocity decreases as particles approach the bottom of the cavity $(@ \mathrm{Z}=0 \mathrm{~cm})$. Also, a triangular shape of low to zero velocity is formed from the bottom to the top and disappears into the zone near the static walls. The solid particles do not move in the mentioned 
triangular region due to the consolidation process of the granular material produced by the increase of the normal stress and the shear stress of the floor of the mixer, resulting in a poor mixing product. These results were also found in the experiments performed by Obregón et al., [4].

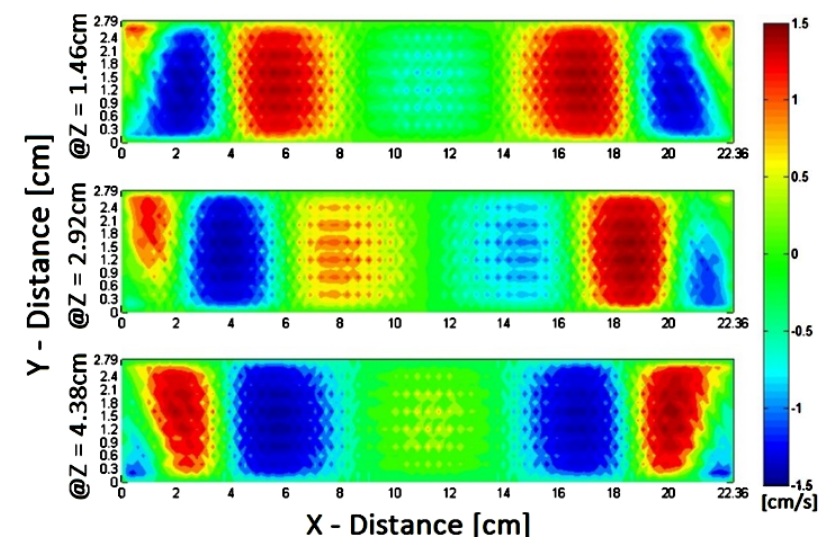

Fig. 8. Profile of the Z-velocity at different heights

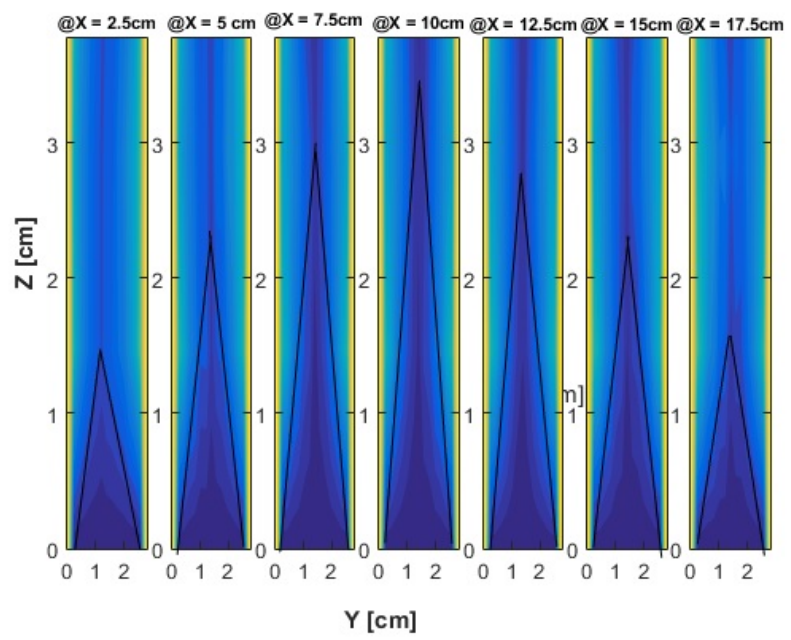

Fig. 9. Profile of the total velocity at different lengths

Fig. 10 shows the total velocity profiles in the $\mathrm{XZ}$ plane at different $\mathrm{Y}$. In the zone at $\mathrm{Y}=\mathrm{L} / 2$, the velocity is low, while in the regions close to the oscillatory walls, located at $\mathrm{Y}=0 \mathrm{~cm}$ and $\mathrm{Y}=2.79 \mathrm{~cm}$ the velocity profiles reach the maximum because of the shear stresses produced by the movement of the particles next to the moving wall.

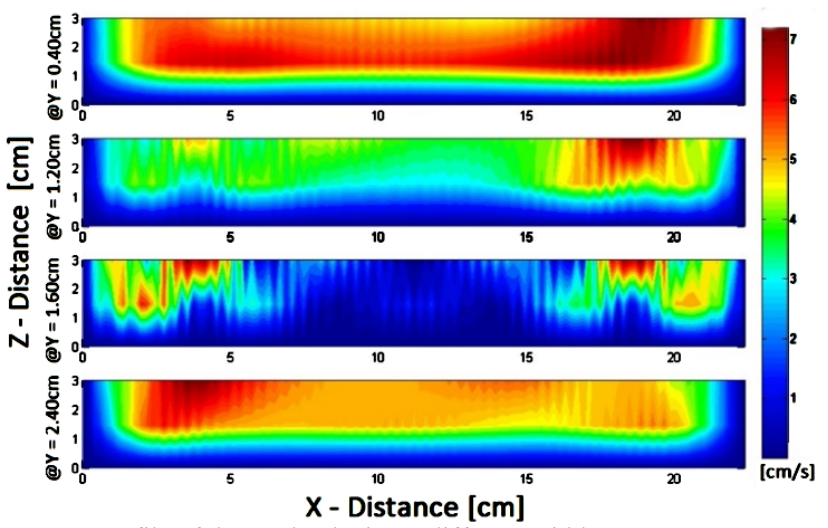

Fig. 10. Profile of the total velocity at different widths

Regarding the volumetric fraction of the powder, as shown in Fig. 11, the concentration of the solid in the central zone of the plane present small variations different than in the corners of the cavity where the variation reaches the maximum and minimum volumetric fractions. In general, the granular material experienced a slight continuous expansion and an increase in the volume fraction in the central zone from 0.54 to 0.55 vol. $\%$ due to the consolidation process. The movement of the powder in the proximities of the walls is affected by the volumetric fraction of the granular material, and this is why, the $\mathrm{X}$-velocity profile present maximum values in the region near the corners, where the volumetric fractions are the highest [29].

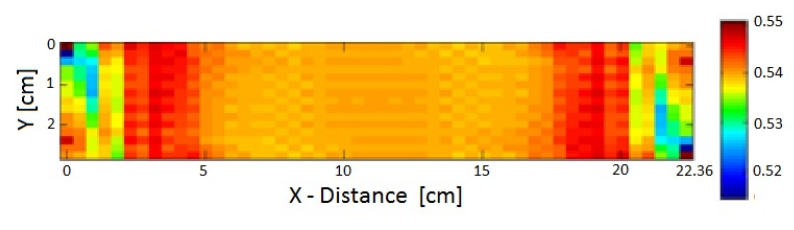

Fig. 11. Profile of the volumetric fraction at $Z=2.52 \mathrm{~cm}$

\subsection{Statements about stability}

In the conditions for the stable response, the time of simulation was too big. Errors introduced by the method of finite differences were first order with respect to time increments. A semi-implicit method is recommended to give a solution to the optimization problem. Implementing the finite volume method with the use of the non-iterative PISO (Pressure-Implicit with Splitting of Operators) algorithm [30] is an excellent alternative to obtain stable results for more extended periods of simulation. The PISO method is faster than iterative techniques for transient flow, such as SIMPLE and SIMPLER [31], stable for large time-steps making it efficient for steady-state as well as transient state, and applicable to compressible flow régimes. The PISO method uses the splitting of operations in the solution of the discretized momentum and pressure equations and the results obtained at each time-step are close approximations to the exact solution of the partial differential equations system.

\section{Validation and Verification}

In this paper, a qualitative validation of the computer simulation model was made by comparing the real distribution of the particles in the plane $Y Z$ from the experiment results carried out by Obregon et al. [4], with the velocity profile found through simulation under the same condition of the experiment. The triangular shape region with no motion of particles was found in both, simulation (see Fig. 9) and experiment. It means that the mathematical model chosen to simulate the powder behavior inside the mixer is accurate.

The tendency of the maximum height of the triangular behavior presented in the plane $\mathrm{YZ}$ along the $\mathrm{X}$-direction obtained experimentally $\left(\mathrm{h}_{\mathrm{e}}\right)$ was compared to the obtained with the simulation results $\left(h_{s}\right)$, see Fig. 12. The average relative errors were around $27.8 \%$ with a standard deviation of 0.4 , indicating low exactitude but good precision. This situation can be corrected by adjusting the model and choosing a better solution method for the simulation, with the aim of minimizing the total error. Also, a strong correlation was found between the variables shown in Fig. 13 , where the $\mathrm{R}^{2}$ coefficient was 0.98 approximately.

The code developed in MATLAB $\AA$ corresponding to the method of solution of the equations of continuity, 
momentum and stress tensor was run to simulate the viscous flow of glycerin in the oscillatory blender, under the same conditions in which the simulation of the granular material was performed. The same simulation with the same parameters was run in the software STAR-CCM+.

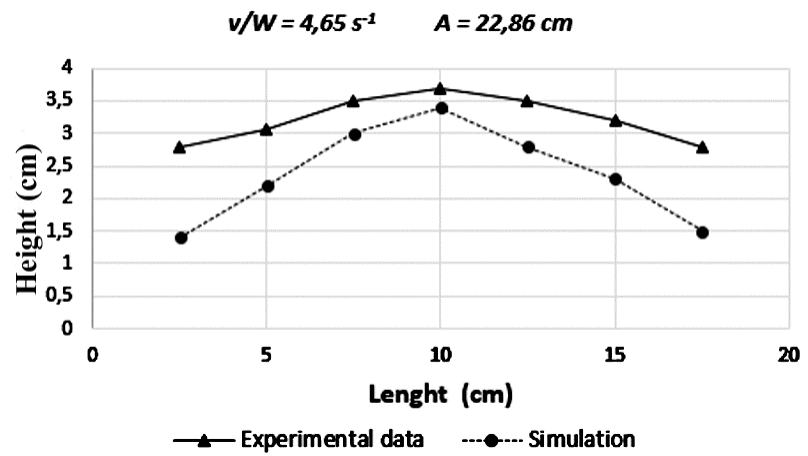

Fig. 12. Tendency of the maximum height of the triangular behavior

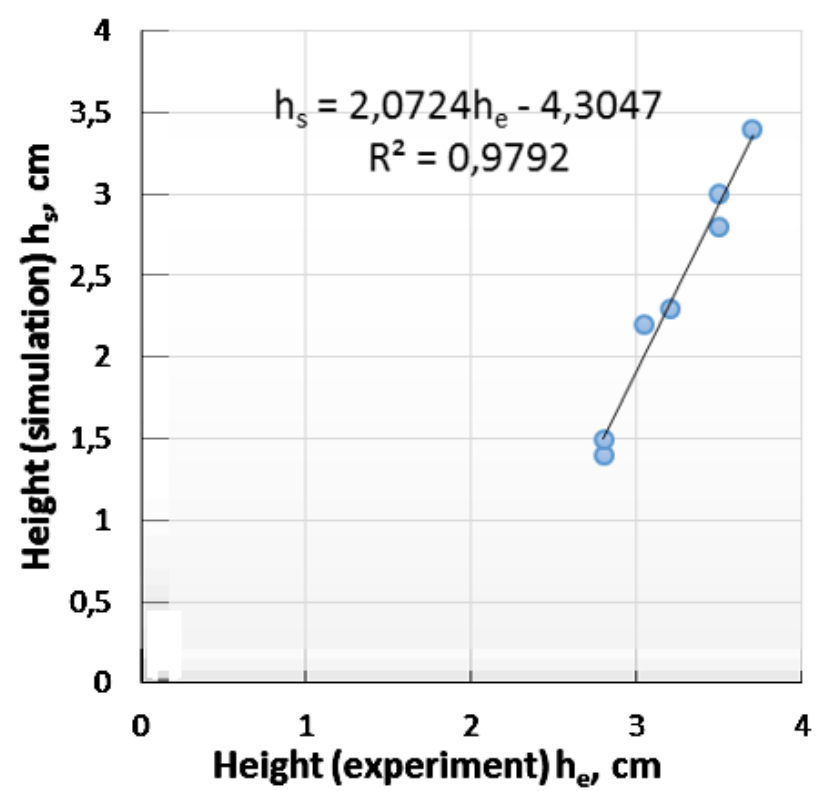

Fig. 13. Correlation between experimental data and simulation, height of triangular behavior

The results of the simulations in both programs were compared using the $\mathrm{X}$-velocities in a plane XY, parallel to the free surface of the fluid, see Fig. 14. It can be seen that the profiles obtained using both software are highly similar.
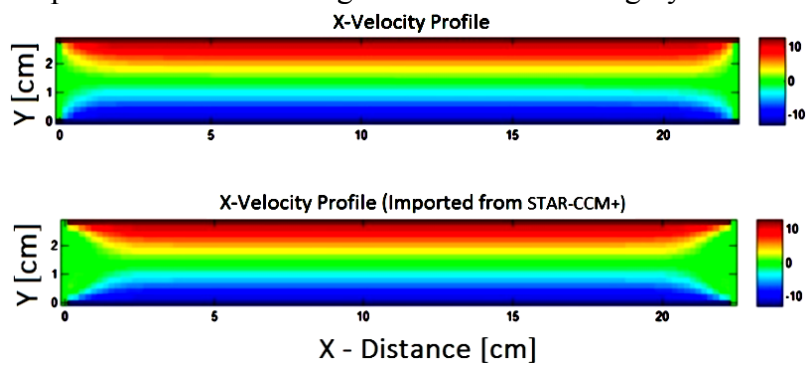

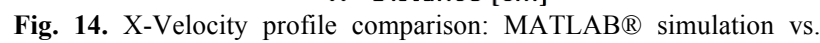
STAR-CCM+

Fig. 15 shows the absolute error obtained with the difference in X-velocity using the code in Matlab ${ }^{\circledR}$ and STAR-CCM+. It can be seen that $83.5 \%$ of the data had an error lower than $0.5 \mathrm{~cm} / \mathrm{s}$. The rest of the data had errors lower than $5 \mathrm{~cm} / \mathrm{s}$.

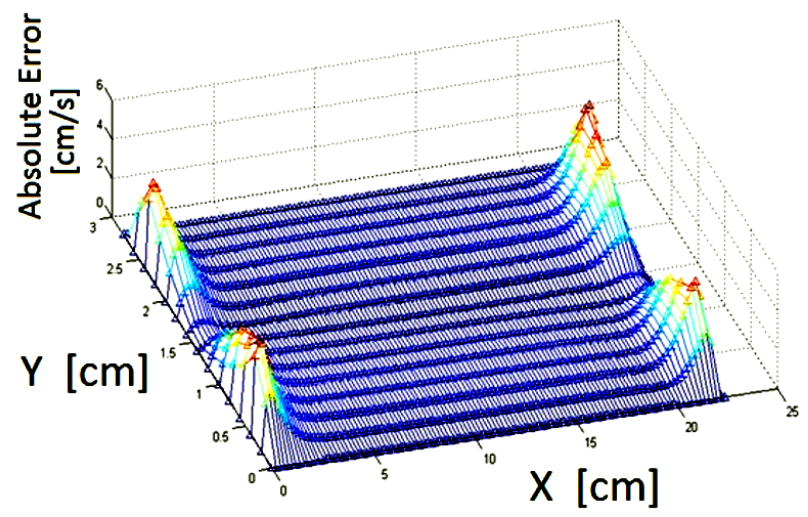

Fig. 15. Absolute error scalar field

The presence of errors happened because the code written in Matlab considered equations that take into account the volume fraction necessary for granular material which is higher in the corners.

\section{Conclusions}

- The simulation of the flow of powder when shear stress was applied and assuming non-Newtonian fluid using the finite differences method under the explicit scheme was achieved, finding the velocity profiles and the volume fraction of solid for different planes. The results of the simulation are coherent and similar to the experimental results found in the literature.

- It was found a region inside the cavity of the blender where the velocity was almost zero due to the consolidation of the solid. It was caused because of the increase of the normal stress at the bottom of the mixer.

- The vortices presented due to the variation of the volumetric fraction of the powder, i.e., dilatation, make possible the mixing of the particles in the $\mathrm{Z}$ direction.

- The finite difference method under the explicit scheme presents a small range of stability, making very impractical to perform simulations for long periods of time.

- The program code was verified comparing the simulation results with the STAR-CCM + software, and it was found that the $83.5 \%$ of the compared data showed absolute errors lower than $0.5 \mathrm{~cm} / \mathrm{s}$.

\section{Acknowledgments}

The authors are very grateful to Universidad del Atlántico and COLCIENCIAS through the financial aid given to the project and with the help of the University of Puerto Rico at Mayagüez with the software used.

This is an Open Access article distributed under the terms of the Creative Commons Attribution Licence

\section{References}


1. F. Bertrand, L.A. Leclaire, G. Levecque, Chem. Eng. Sci. 60(8-9), p. 2517-2531 (2005).

2. L. Quiñones, C. Velazquez, L. Obregon, AIChE J. 60(9), p. 31233132 (2014).

3. F.J. Muzzio, T. Shinbrot, B.J. Glasser, Powder Technol. 124(1-2), p. 1-7 (2002).

4. L. Obregón, A. Realpe, C. Velázquez, Powder Technol. 197(1-2), p. 9-16 (2009).

5. W.R. Ketterhagen, Powder Technol. 275(0), p. 361-374 (2015).

6. R.P. Zou, A.B. Yu, Powder Technol. 88(1), p. 71-79 (1996).

7. H.J. Venables, J.I. Wells, Drug Devel. Ind. Pharm. 27(7), p. 599612 (2001).

8. P.J. Abrahamsson, S. Sasic, A. Rasmuson, Powder Technol. 268(0), p. 339-346 (2014).

9. P.C. Johnson, R. Jackson, J. Fluid Mech. 176, p. 67-93 (1987).

10. P. Jop, Y. Forterre, O. Pouliquen, Nature 441(7094), p. 727-730 (2006).

11. C. Varsakelis, M.V. Papalexandris, J. Comput. Phys. 257 Part A(0), p. 737-756 (2013)

12. W.-T. Wu, N. Aubry, M. Massoudi, Mech. Res. Comm. 52(0), p. 62-68 (2013).

13. M. Massoudi, Int. J. Non-Linear Mech. 38(3), p. 313-336 (2003).

14. M. Massoudi, Mech. Res. Comm. 35(8), p. 603-608 (2008).

15. M. Massoudi, G. Ahmadi, Int. J. Non-Linear Mech. 29(4), p. 487492 (1994).

16. M. Massoudi, E.J. Boyle, Int. J. Non-Linear Mech. 36(4), p. 637648 (2001).
17. H. Zhu, M.M. Mehrabadi, M. Massoudi, Int. J. Plast. 22(5), p. 826857 (2006).

18. H. Zhu , M.M. Mehrabadi, M. Massoudi, Comp. Math. Appls. 53(2), p. 244-259 (2007).

19. J. Baxter, H. Abou-Chakra, U. TüZün, B. M. Lamptey, Chem. Eng. Res. Des. 78(7), p. 1019-1025 (2000).

20. E.D. Fernández-Nieto, J. Garres-Díaz, A. Mangeney, G. NarbonaReina, J. Comput. Phys. 356, p. 192-219 (2018).

21. X. Zhao, M. Massoudi, Appl. Math. Comput. 242, p. 518-527 (2014).

22. L. Obregón, A. Realpe, and C. Velázquez, Powder Technol. 201(3), p. 193-200 (2010).

23. C.S. Campbell, Powder Technol. 162(3), p. 208-229 (2006).

24. D.G. Schaeffer, J. Diff. Eqns. 66(1), p. 19-50 (1987).

25. S. Dartevelle, Geochemistry, Geophysics, Geosystems 5(8), p. 1-28 (2004).

26. D. Gray, J. Stiles, Topical Report DOE/MC/21353-2584 (DE88001089), U.S. Department of Energy (1988).

27. S.A. Elaskar, L.A. Godoy, D.D. Gray, J.M. Stiles, In Int. J. Solids Stru. 37(15), p. 2185-2214 (2000).

28. G.D. Smith, Oxford University Pess (1985)

29. Z. Xiao Ming, J.M. Hill, Appl. Math. Modelling, 20(1), p. 82-92 (1996).

30. R.I. Issa, J. Comput. Phys. 62(1), p. 40-65 (1986).

31. H.K.Versteeg, W. Malalasekera, Pearson Education Limited (2007). 